\title{
Counter Cross-Cultural Priming and Relative Deprivation: The Role of Individualism-Collectivism
}

\author{
Kees van den Bos • Tanja S. van Veldhuizen • \\ Al K. C. Au
}

Published online: 23 December 2014

(C) Springer Science+Business Media New York 2014

\begin{abstract}
This paper uses cross-cultural comparisons and comparisons obtained by experimental manipulation to examine how cultural and contextual factors influence responses to personal and group relative deprivation. Two studies were conducted, one in an individualistic country (The Netherlands) and one in a collectivistic country (Singapore). One way to examine the influence of the assumed cultural differences in individualism-collectivism is to assign participants to the conditions that elicit "countercultural" psychological states, that is, conditions that prime collectivistic mindsets in the Netherlands and individualistic mindsets in Singapore. Results show that cross-cultural differences have reliable effects on responses to relative deprivation and gratification. Furthermore, findings in the countercultural (experimental) conditions meaningfully differed from those observed in the control conditions in which participants were exposed to neutral stimulus materials. This suggests that cultural mindsets are not fixed, and that countercultural priming can be used to study cross-cultural and contextual differences with high levels of internal validity.
\end{abstract}

An earlier version of this article was submitted to Utrecht University as Tanja van Veldhuizen's MSc thesis.

K. van den Bos $(\bowtie)$. T. S. van Veldhuizen

Department of Psychology, Utrecht University, Heidelberglaan 1, 3584 CS Utrecht,

The Netherlands

e-mail: k.vandenbos@uu.nl

Present Address:

T. S. van Veldhuizen

Department of Criminal Law and Criminology, Faculty of Law, Maastricht University, Maastricht, The Netherlands

A. K. C. Au

National University of Singapore, Singapore, Singapore 
Keywords Relative deprivation · Individualism - Collectivism - Cross-cultural differences $\cdot$ Contextual differences $\cdot$ Experimental control $\cdot$ Countercultural priming

Relative deprivation theory describes the responses that are elicited when people feel they are unjustly treated relative to a standard. The experience of relative deprivation has been shown to impact a variety of reactions, such as people's fairness judgments and their intentions to criticize and voice their opinions (e.g., Crosby, 1976; Smith \& Ortiz, 2001; Walker \& Mann, 1987). Furthermore, the standards to which people are responding can consist of various things, such as a similar other person or a comparable other group (Crosby, 1976; Runciman, 1996; Stouffer, Suchman, DeVinney, Star, \& Williams, 1949; Walker, 1999). Imagine, for example, that year-end bonuses are allocated within your company following two possible situations. In the first situation, you get a lower year-end-bonus than your immediate co-worker. In the second situation, all employees in your department get a lower year-end bonus than the employees in another department. In both situations you get the same absolute amount of money thus, objectively, you are equally well off in both situations. Still, research shows that people may react quite differently to these situations (see, e.g., Smith \& Ortiz, 2001; Smith, Pettigrew, Pippin, \& Bialosiewicz, 2012). Similarly, people may respond quite differently when their bonus is better than what other persons versus other groups receive (e.g., Dambrun, Taylor, McDonald, Crush, \& Méot, 2006; Guimond \& Dambrun, 2002; Moscatelli, Albarello, Prati, \& Rubini, 2014).

In the current paper, we examine how people respond to issues of personal deprivation (being disadvantaged as an individual relative to a similar other individual) and group deprivation (being disadvantaged as a group member relative to a similar other group; Walker, 1999). We contrast this with how people respond to issues of personal gratification (being advantaged as an individual relative to a similar other individual) and group gratification (being advantaged as a group member relative to a similar other group). In exploring these issues, we focus on the question of why people sometimes are more sensitive to treatment as an individual whereas in other circumstances they are more sensitive to treatment as a group member. To that end, we note that earlier studies have linked responses to personal and group deprivation to social identity concepts (e.g., Ellemers, 2001; Kawakami \& Dion, 1993; Smith, Spears, \& Oyen, 1994). Specifically, it has been argued that people, whose personal identity is salient in a given situation, are more likely to be sensitive to comparisons that affect them as an individual relative to another individual than to comparisons that affect them as a group member. Therefore, they are thought to be primarily affected by the presence of personal deprivation or personal gratification. In contrast, people whose group membership is salient are assumed to be more sensitive to comparisons that target them as a group member, and are therefore assumed to respond more strongly to issues of group deprivation and group gratification.

Research findings support this reasoning. For example, Kawakami and Dion (1993) primed participants in a college setting such that either strong identification 
with their tutor group was made salient or personal identity was made salient. The authors manipulated whether personal or group deprivation was present or absent. Findings showed that participants whose group membership was salient reported more feelings of group deprivation and stronger intentions to engage in collective action. In contrast, participants whose personal identity was salient were more likely to undertake individual actions against the injustice. Furthermore, Smith et al. (1994) primed psychology students with either a specific group identity or with a personal identity. Then, half of the participants were both personally and group deprived, whereas the other half of the participants were personally gratified but group deprived. Findings obtained revealed that justice judgments about the situation depended on whether their group membership was salient or not.

The studies reviewed suggest that a focus on group membership or the individual identity does influence feelings of group and personal deprivation. Building and extending on these insights, we argue that not only situational variations in personal or group identity influence responses to relative deprivation and relative gratification, but also more chronic inclinations to respond in individualistic or collectivistic terms can impact fairness judgments and intentions to voice opinions following experiences of relative deprivation and relative gratification. In our attempt to provide evidence for this line of reasoning, we link relative deprivation theory to the cultural dimension of individualism versus collectivism (Hofstede, 2001).

\section{Individualism and Collectivism}

One type of chronic focus on the individual or the group is represented by the cultural dimension of individualism versus collectivism. Work on this cross-cultural dimension suggests that in individualistic societies people mainly think in terms of "I" and see themselves as unique individual beings. In contrast, in collectivistic societies people are assumed to think more in terms of "we," thereby primarily viewing themselves in terms of important group memberships (Hofstede, 2001). Accordingly, individualistic societies attach importance to individual initiative, achievement, and autonomy. In these cultures, you are supposed to take care of yourself and to protect your personal goals (e.g., Heine, Markus, Lehman, \& Kitayama, 1999; Hofstede, 2001; Oyserman, 2011; Oyserman, Coon, \& Kemmelmeier, 2002; Sedikides, Gaertner, \& Toguchi, 2003; Triandis, McCusker, \& Hui, 1990). In contrast, collectivistic societies primarily value belonging to a group. Here, it is expected that people invest in social relationships, and fulfill their obligations to the group (e.g., Heine et al., 1999 Hofstede, 2001; Oyserman, 2011; Oyserman et al., 2002; Sedikides et al., 2003; Triandis et al., 1990).

Thus, individualism can be described as a mindset in which personal identities are chronically salient, whereas collectivism can be portrayed as a mindset in which group identities are chronically salient. This led us to expect that people from individualistic and collectivistic cultures should respond differently to personal and group deprivation and/or gratification. Specifically, we expected that personal deprivation and gratification should have a greater impact among participants from individualistic cultures than from participants from collectivistic cultures, whereas 
group deprivation and gratification should be more important in samples obtained in collectivistic cultures than in samples from individualistic cultures.

Importantly, however, our predictions also included contextual effects. That is, we proposed that normally individualists and collectivists respond to personal and group treatment according to mindsets that are the cultural "default." However, when mindsets are made salient that are opposite to the culturally default mindsets; this will probably have an influence on their reactions in the direction of the salient contextual mindsets. Thus, we argued that it is possible to manipulate cultural mindsets and to push people's responses to relative deprivation and relative gratification in countercultural directions.

Although this position might seem controversial (cf. Heine et al., 1999), it is consistent with a view on culture as a flexible and dynamic system, rather than a fixed set of stable characteristics (see, e.g., Hong, 2009; Hong \& Chiu, 2001; Kühnen, Hannover, \& Schubert, 2001; Matsumoto \& Hee Yoo, 2006; Miller, 2002; Oyserman, 2011; Oyserman \& Lee, 2008; Van den Bos et al., 2010; Van den Bos, Brockner, Van den Oudenalder, Kamble, \& Nasabi, 2013). Specifically, cultural expressions may be malleable by context and meaning that whether people adhere to mindsets that are default in their culture actually depends on the situation that they are in (e.g., Oyserman, 2011; Oyserman \& Lee, 2008; Van den Bos et al., 2010, 2013). As long as a default cultural mindset is valued and expected in a given situation, people's reactions to personal and group treatment will reflect responses to this pro-cultural mindset. However, if countercultural mindsets are made salient, people's reactions might be affected significantly by these countercultural mindsets.

The idea behind our position is that mindsets belonging to the cultural system that people grow up in are easily accessible. Yet, in the modern world, people do have knowledge of norms and mindsets that are opposite of their default cultural mindsets. These latter countercultural mindsets can be made salient through contextual priming. For example, a collectivist who normally focuses on a duty to the ingroup, might be asked to think of his or her personal uniqueness and personal expectations. Research shows that, as a result of this task, personal goals become salient and individualistic mindsets are temporarily induced (Oyserman \& Lee, 2007; Trafimow, Triandis \& Goto, 1991). Consequentially, the person who engaged in the individual priming task is likely to perceive the social world through a more individualistic perspective and respond in a countercultural way (Oyserman \& Lee, 2007).

Thus, we hypothesized that both cultural differences and contextual differences can affect responses to personal and group deprivation and/or gratification. Normally, people interpret a situation from the perspective of their chronically available cultural mindset. Consequently, individualists should be more sensitive to personal deprivation and personal gratification, whereas collectivists should be more sensitive to group deprivation and group gratification. However, we expected that if countercultural mindsets are salient in the social context, participants from individualist cultures will adhere more strongly to collectivistic mindsets, whereas participants from collectivist cultures will respond in more individualistic ways (compared to when countercultural mindsets are not salient). We studied these effects using a countercultural priming paradigm. 


\section{Studying Cross-Cultural Differences with a Countercultural Priming Paradigm}

A nagging concern when studying cross-cultural differences is that one often has to refer to samples from cultures that are conveniently accessible. Furthermore, in many cross-cultural studies there often is not enough empirical evidence for the assumed psychological mechanisms (Brockner, 2003). That is, frequently crosscultural research studies include people from two or more countries and assume that the research participants differ on certain psychological dimensions. These differences on psychological dimensions are then assumed to affect subsequent reactions of the participants. As noted by Van den Bos et al. (2013), if the research findings suggest that the participants in the different countries react differently, and that these reactions are in accordance with the psychological dimensions presumed to differ across cultures, it is still necessary to provide evidence that the culturally varying reactions can be attributed to the hypothesized psychological dimensions.

Researchers sometimes try to provide such evidence by measuring the relevant psychological dimensions, and then use tests of mediation to asses whether the cultural differences were attributable to the psychological dimensions (Van den Bos et al., 2013). However, measuring the hypothesized mediating psychological dimensions may be difficult (see, e.g., Spencer, Zanna, \& Fong, 2005). For example, the psychological dimensions may refer to unconscious processes that are difficult to assess. Relatedly, measuring psychological dimensions in cross-cultural research often involves self-reports, which may be problematic because of people's unwillingness or inability to disclose their true beliefs (e.g., Nisbett \& Ross, 1980). Furthermore, a well-known issue in cross-cultural studies is that measures of the same construct may not mean the same thing to people from different cultures (Hui \& Triandis, 1985; Matsumoto, 2003). In short, there may be important problems associated with measuring the psychological dimensions presumed to account for cross-cultural differences. In addition, even when it is possible to measure reliably the hypothesized psychological mediators of the effects of culture, the ensuing statistical analyses can at best provide only correlational evidence which limits the empirical strength of the study in question (Van den Bos et al., 2013).

In the present paper, we argue that there is a need in cross-cultural research to not only provide evidence of why people from different cultures show different reactions, but also to do so in ways that entail a high degree of internal validity (Van den Bos et al., 2010). In fact, for purposes of internal validity an experimental approach in which participants are randomly assigned to conditions differing along the psychological dimension hypothesized to account for the results may have important advantages. For example, one of the virtues of such an approach is that it is likely to hold constant all factors other than the construct being manipulated. As a result, differences that emerge on dependent variables are likely to have been causally influenced by the manipulated construct (Van den Bos et al., 2013). In the current paper, we employ such an experimental approach to cross-cultural research on relative deprivation and relative gratification using a method in which we prime (vs. do not prime) countercultural mindsets among people from individualistic and collectivistic cultures, and we assess how the resulting variations in culture (i.e., 
country) and context (i.e., experimental condition) impact participants' reactions to being deprived or advantaged as an individual person or as a member of a group.

There is growing evidence for the possibility of priming culture (see, e.g., Bechtoldt, Choi, \& Nijstad, 2012; Bechtoldt, De Dreu, Nijstad, \& Choi, 2010; Kühnen et al., 2001; Kühnen \& Oyserman, 2002; Oyserman \& Lee, 2008; Van den Bos et al., 2010, 2013). Earlier studies that explicitly used a countercultural priming paradigm did so in the context of differences in masculinity-femininity (Van den Bos et al., 2010) and power distance (Van den Bos et al., 2013) and focused on responses to being allowed or denied opportunities to voice one's opinions. Relative deprivation obviously plays a very important role in people's lives across this world and so do differences in individualism and collectivism. As noted by Baumeister (2005, p. 177): "Today, the vast majority of cultural psychologists focus on comparing Eastern and Western cultures, because these are the most reliably different." Thus, we think the studies to be presented here are important for both the study of cross-cultural differences and relative deprivation.

\section{The Current Research}

Two studies are presented in the current paper. Study 1 was executed in The Netherlands and Study 2 took place in Singapore. The Netherlands score high on the individualism versus collectivism dimension (i.e., 80; Hofstede Centre, 2013), suggesting that the Dutch normally tend to be focused on their personal state of affairs and primarily think of themselves in terms of "I" (Hofstede, 2001). Singapore, on the other hand, scores low on this dimension (i.e., 20; Hofstede Centre, 2013), indicating that Singaporeans tend to be oriented toward the group and are likely to think in terms of "we" (Hofstede, 2001).

Thus, the Netherlands and Singapore are at opposite ends of the individualism versus collectivism dimension. This is not to say that no other cultural dimensions could distinguish the Netherlands and Singapore, but the clear distinction in individualism and collectivism between the two countries helped us when operationalizing the countercultural priming manipulation that we induced in both studies presented here. That is, building on earlier studies that used a countercultural priming paradigm (Van den Bos et al., 2010, 2013), in both our studies half of the participants were primed with stimuli that have been shown in earlier studies to be perceived in neutral ways by research participants and that we assumed would keep their chronically accessible cultural mindset intact. We expected that in this control condition-in which countercultural priming was absent-research participants would adhere to mindsets that can be assumed to be default and belonging to their national culture. In contrast, the other half of the participants were presented with countercultural primes. Specifically, using the conceptualization by Trafimow et al. (1991), we constructed individualistic and collectivistic primes. The Dutch participants in the countercultural or experimental condition of Study 1 responded to a collectivistic prime (Trafimow et al., 1991), and the Singaporean participants in the countercultural or experimental condition of Study 2 responded to an individualistic prime (Trafimow et al., 1991). We predicted that when people had 
been (vs. had not been) brought in these countercultural mindsets their reactions would adhere more strongly to norms and values that are normally not associated with their cultural background (Van den Bos et al., 2010, 2013).

Because our experimental priming manipulations were designed to capture specifically the individualism versus collectivism dimension (Trafimow et al., 1991), we argued that we could attribute participants' reactions following the priming manipulations to this dimension. We used this insight to assess how our participants responded to outcomes in terms of fairness judgments and their intentions to voice their opinions. In all the conditions, participants received the same outcome and this outcome was unfair in all the conditions. We varied those standards to which participants responded such that when participants were deprived their outcome was worse than the outcome of others, whereas when participants were advantaged their outcome was better than the outcome of others. We also varied that participants were affected either as an individual relative to other individuals or as a member of a group relative to another group. We predicted that when an individualistic norm was salient, participants' ratings of fairness and willingness to criticize a decision would be influenced by their personal advantage or disadvantage. We predicted that when a collectivistic norm was salient, participants' ratings of fairness and willingness to citizen would be influenced by their group's advantage or disadvantage.

\section{Study 1}

\section{Method}

Participants and Design Two hundred and thirty-nine students at Utrecht University in the Netherlands participated in Study 1 (75 men, 164 women; $M_{\text {age }}=20.86, \mathrm{SD}_{\mathrm{age}}=2.25$ ). Participants were randomly assigned to one of the conditions of a 2 (countercultural priming: absent vs. present) $\times 2$ (deprivation: deprived vs. advantaged) $\times 2$ (level of treatment: personal vs. group) betweensubjects design.

Experimental Procedure Students were invited to participate in the paper-andpencil experiment in designated study areas or university canteens. Participants took part on a voluntary basis and were not paid for their participation. The questionnaire that made up the experiment consisted of four parts: a priming manipulation; a filler task; the relative deprivation manipulation with dependent variables; and an assessment of demographics. Participants were quite fluent in English and all materials were written in English in an attempt to control language effects between Studies 1 and 2.

The questionnaire started with the priming manipulation. The condition in which countercultural priming was present to our Dutch participants was modeled directly after the collectivistic prime of the Similarities and Differences with Family and Friends (SDFF) task (Trafimow et al., 1991). Accordingly, participants in this 
condition were informed that recent research has shown that people's thoughts about their friends and family tell a lot about them and that we therefore asked them to think about their friends and family and to complete the following two questions to that effect: (1) "Please think for a minute about what you have in common with your family and friends. How are you similar to each other?," and (2) "What do your family and friends generally expect you to do?." Similar primes have been shown to effectively alter cultural mindsets (Oyserman \& Lee, 2007).

The condition in which countercultural priming was absent was modeled after stimulus materials that served successfully as control primes in various earlier studies (see, e.g., Greenberg, Solomon, \& Pyszczynski, 1997). Specifically, participants were told that recent research has shown that people's thoughts about watching television tell a lot about them and that we therefore asked them to think about watching television and complete the following two questions related to this issue: (1) "Please think for a minute about watching television. How would you describe a typical moment of watching television?," and (2) "With what expectations do people generally watch television?." Earlier research suggests that these kinds of questions are perceived in neutral ways by participants and leave their default mindsets intact (e.g., Loseman, Miedema, Van den Bos, \& Vermunt, 2009; Van den Bos, 2001; Van den Bos et al., 2012, 2013; Van den Bos \& Miedema, 2000; Van Prooijen, Van den Bos, \& Wilke, 2002).

To let the priming manipulation sink in, participants continued by filling out the Positive And Negative Affective Schedule (PANAS; Watson, Clark, \& Tellegen, 1988). In line with earlier research (e.g., Greenberg et al., 1997; Loseman et al., 2009; Van den Bos, 2001; Van den Bos et al., 2012, 2013; Van den Bos \& Miedema, 2000; Van Prooijen et al., 2002), this scale served as a filler task. The scale consists of 10 items measuring positive affective states $(\alpha=.86)$ and 10 items measuring negative affective states $(\alpha=.87){ }^{1}$

After filling out the PANAS, both deprivation and level of treatment were manipulated. To this end, participants were asked to read and respond to scenarios about the allocation of year-end bonuses. In all conditions, the scenarios started with the following introduction.

Imagine that you are working as a consultant at a medium-sized company. The company has two similar departments that are only slightly different in the clients they serve. Each department holds approximately 15 persons. Your

\footnotetext{
1 Please note that after the prime manipulation and before reading the scenarios (which included the deprivation and level of treatment manipulations), participants completed the 20 items that, combined, constitute the PANAS (Watson et al., 1988). The PANAS items asked participants to indicate their present affective states. Importantly, after having read the scenarios (and hence having completed all manipulations), participants completed different questions. Specifically, participants then were asked to answer 24 questions about the situation portrayed in the scenarios (see Van Veldhuizen, 2013). We included the PANAS in our studies because earlier research shows that priming manipulations that use open-ended questions to induce mindsets have stronger effects when followed by filler tasks. Thus, as in earlier studies (e.g., Greenberg et al., 1997; Loseman et al., 2009; Van den Bos, 2001; Van den Bos et al., 2012, 2013; Van den Bos \& Miedema, 2000; Van Prooijen et al., 2002), the PANAS served as a filler task in our studies and (as mentioned in Footnotes 2 and 4) controlling for positive and negative affective states as assessed by the PANAS did not alter the findings reported. Therefore, the effects of affective states are not reported further. Data and stimulus materials are available on request.
} 
position is in one of the two departments. So far, you feel valued by the management. Furthermore, the secondary benefits are very good, so you are pretty happy about your position. Every year the company awards a year-end bonus to both departments, to thank them for their hard work. Thus, when the end of the year is coming into sight, you already start thinking about how to spend the bonus this year.

In each condition, the scenario ended with two sentences in which all participants were informed that they received a year-end bonus of $€ 500$. Thus, the year-end bonus amount was always the same. The sentences were set up in such a way that deprived participants received a lower year-end bonus than others, whereas advantaged participants received a higher year-end bonus than others. Level of treatment was manipulated such that participants were affected either as a member of the department relative to the other department or affected as an individual worker relative to their immediate co-workers. Specifically, when participants were group deprived the scenario ended as follows:

Without warning or explanation, the management reveals that this year the bonus will be different for each department. Employees in your department will receive $€ 500$ this year, whereas the employees in the other department will receive $€ 1000$.

Participants who were personally deprived read the following two sentences:

Without warning or explanation, the management reveals that this year the bonus will be different for each employee. You will receive $€ 500$ this year, whereas your 14 immediate co-workers each will receive $€ 1000$.

Participants who were group advantaged read the following:

Without warning or explanation, the management reveals that this year the bonus will be different for each department. Employees in your department will receive $€ 500$ this year, whereas the employees in the other department will receive $€ 100$.

Finally, participants who were personally advantaged read:

Without warning or explanation, the management reveals that this year the bonus will be different for each employee. You will receive $€ 500$ this year, whereas your 14 immediate co-workers each will receive $€ 100$.

We report all manipulations, all data exclusions, and all measures in our studies (Simmons, Nelson, \& Simonsohn, 2012), so we note that the scenarios were followed by 24 items measuring different categories of reactions such as fairness judgments, intentions to voice opinions, and positive and negative feelings about treatment in the scenarios. All questions were answered on 7-point scales $(1=n o t$ at all, $7=$ very much) and univariate analyses revealed that participants of Study 1 responded strongest in terms of our predictions to the following three items: "How fair do you think the $€ 500$ bonus is?," "How fair do you feel you are treated?," and "To what extent do you feel that the $€ 500$ is right?" Participants' answers to these 
questions constituted a reliable fairness judgments scale $(\alpha=.79)$. Additionally, we also included in our analyses the items that yielded the strongest effects in the Singaporean sample of Study 2 and that constituted a reliable scale of participants' individual intentions to voice their opinions in Study $1(\alpha=.75)$. "To what extent would you like to express your opinion about the $€ 500$ bonus?," and "To what extent would you like to criticize the management of the company?." The way in which these two scales were constructed will be discussed in the General Discussion (see also Van den Bos et al., 2012). Table 1 shows the grand means, standard deviations, and correlations regarding the dependent variables of Studies 1 and 2. Tables 2 and 3 present the means and standard deviations within each cell of the two studies.

\section{Results}

Fairness Judgments A 2 (countercultural priming: absent vs. present) $\times 2$ (deprivation: deprived vs. advantaged) $\times 2$ (level of treatment: group vs. personal) analysis of variance on the fairness judgments scale revealed a main effect of deprivation, $F(1,222)=11.57, p<.002, \eta^{2}=.04$, an interaction effect between deprivation and level of treatment, $F(1,222)=9.97, p<.01, \eta^{2}=.04$, and the predicted three-way interaction effect between countercultural priming, deprivation, and level of treatment, $F(1,222)=5.11, p<.03, \eta^{2}=.02 .{ }^{2}$ Other effects were not statistically significant, $p s>.31$.

Figure 1 shows the three-way interaction effect. To interpret this effect, it is relevant to note that in the condition in which countercultural priming was absent we obtained a significant two-way interaction effect between deprivation and level of treatment, $F(1,222)=14.64, p<.001, \eta^{2}=.06$. This effect indicated that, in the absence of countercultural priming, our Dutch participants found it less fair to be personally deprived $(M=2.40, \mathrm{SD}=1.06)$ than to be group deprived $(M=3.08$, $\mathrm{SD}=0.97), F(1,222)=4.97, p<.03, \eta^{2}=.02$. Furthermore, participants found it more fair to be personally advantaged $(M=3.55, \mathrm{SD}=1.24)$ than to be group advantaged $(M=2.63 \mathrm{SD}=1.08), F(1,222)=9.76, p<.003, \eta^{2}=.04$. Viewed differently, in the absence of countercultural priming, the Dutch participants found

\footnotetext{
2 Inspecting Cook's (1977) distance measure in this analysis (Cohen, Cohen, West, \& Aiken, 2003) revealed that 7 of the 239 participants of Study 1 (2.9\% of the sample) showed a distance score of more than 3 SDs above the mean. These participants were excluded from the main analyses of Study 1 . Two other participants $(0.8 \%$ of the sample) were also excluded because of missing values on the fairness judgments scale, leaving a total of 230 participants, with 27-30 participants in each cell of our design. Although the answers of at least some of the excluded participants may have deviated from what was expected, including them in our analyses yielded a similar three-way interaction effect between countercultural priming, deprivation, and level of treatment on participants' fairness judgments as is reported in the main text, although in this case the effect was marginally significant only, $F(1$, $229)=3.44, p=.065, \eta^{2}=.01$. It also should be noted that when controlling for positive and negative affective states as assessed by the PANAS, the predicted three-way interaction was still statistically significant, $F(1,189)=4.58, p<.04, \eta^{2}=.02$. Therefore, the effects of affective states are not reported further in Study 1.
} 
Table 1 Grand means, standard deviations, and correlations regarding the dependent variables of Studies 1 and 2

\begin{tabular}{llll}
\hline & $M$ & SD & $r$ \\
\hline Study 1 & & & $-.37^{*}$ \\
$\quad$ Fairness judgments & 3.02 & 1.26 & 1.57 \\
$\quad$ Voice intentions & 4.87 & & $-.54^{*}$ \\
Study 2 & & 1.39 & 1.59 \\
$\quad$ Fairness judgments & 3.32 & 4.31 & \\
Voice intentions & & \\
\hline
\end{tabular}

The correlation coefficients reported are correlations between participants' fairness judgments and their intentions to voice their opinions. * $p<.001$

it more fair to be personally advantaged than to be personally deprived, $F(1$, $222)=14.98, p<.001, \eta^{2}=.06$, and found it equally unfair to be deprived and to be advantaged when they were affected as a group, $F(1,222)=2.30, p>.13$, $\eta^{2}=.01$.

In the condition in which countercultural priming was present, the interaction effect between deprivation and level of treatment was not statistically significant, $F(1,222)=0.40, p>.52, \eta^{2}=.00$. In this condition, there was only a significant main effect of deprivation, $F(1,222)=9.70, p<.003, \eta^{2}=.04$, indicating that, in the presence of countercultural priming, our Dutch participants found it less fair to be deprived $(M=2.61, \mathrm{SD}=1.00)$ than to be advantaged $(M=3.27, \mathrm{SD}=1.26)$.

Voice Intentions A 2 (countercultural priming) $\times 2$ (deprivation) $\times 2$ (level of treatment) analysis of variance on participants' intentions to voice their opinions revealed a significant main effect of the deprivation manipulation only, $F(1$, $221)=6.88, p<.01, \eta^{2}=.03 .{ }^{3}$ Participants were inclined to voice more against being deprived $(M=5.20, \mathrm{SD}=1.31)$ than against being advantaged $(M=4.68$, $\mathrm{SD}=1.68)$. The three-way interaction of priming, deprivation, and level of treatment was not statistically significant, $F(1,221)=0.77, p>.37, \eta^{2}=.00$. Other effects in the $2 \times 2 \times 2$ analysis were also not statistically significant, $p s>.11$.

\section{Discussion}

The results we obtained on participants' fairness judgments are in line with the view that people from an individualistic country such as the Netherlands are normally focused on their personal state of affairs such that, in the absence of countercultural priming, our Dutch participants found it less fair to be deprived as an individual than to be deprived as a member of a group, whereas they found it more fair to be advantaged as an individual than to be advantaged as a member of a group. When

\footnotetext{
3 One participant had a missing value on one of the voice items, explaining the degrees of freedom reported.
} 
Table 2 Cell means and standard deviations of fairness judgments and voice intentions among Dutch participants as a function of the absence versus presence of the priming of a collectivistic mindset and being deprived or advantaged as a group or person (Study 1)

\begin{tabular}{|c|c|c|c|c|c|c|c|c|}
\hline \multirow[t]{3}{*}{ Dependent variable } & \multicolumn{4}{|c|}{ Countercultural priming absent } & \multicolumn{4}{|c|}{ Countercultural priming present } \\
\hline & \multicolumn{2}{|c|}{ Deprived } & \multicolumn{2}{|c|}{ Advantaged } & \multicolumn{2}{|c|}{ Deprived } & \multicolumn{2}{|c|}{ Advantaged } \\
\hline & $M$ & SD & $M$ & SD & $M$ & SD & $M$ & SD \\
\hline \multicolumn{9}{|l|}{ Fairness judgments } \\
\hline Group treatment & 3.08 & 0.97 & 2.63 & 1.08 & 2.64 & 0.93 & 3.16 & 1.35 \\
\hline Personal treatment & 2.40 & 1.06 & 3.57 & 1.26 & 2.58 & 1.08 & 3.37 & 1.19 \\
\hline \multicolumn{9}{|l|}{ Voice intentions } \\
\hline Group treatment & 5.28 & 1.03 & 4.67 & 1.59 & 5.09 & 1.21 & 4.72 & 1.74 \\
\hline Personal treatment & 4.82 & 1.74 & 4.48 & 1.71 & 5.61 & 1.13 & 4.82 & 1.74 \\
\hline
\end{tabular}

Means are on 7-point scales with higher values indicating more positive fairness judgments and stronger voice intentions, respectively

Table 3 Cell means and standard deviations of fairness judgments and voice intentions among Singaporean participants as a function of the absence versus presence of the priming of an individualistic mindset and being deprived or advantaged as a group or person (Study 2)

\begin{tabular}{|c|c|c|c|c|c|c|c|c|}
\hline \multirow[t]{3}{*}{ Dependent variable } & \multicolumn{4}{|c|}{ Countercultural priming absent } & \multicolumn{4}{|c|}{ Countercultural priming present } \\
\hline & \multicolumn{2}{|c|}{ Deprived } & \multicolumn{2}{|c|}{ Advantaged } & \multicolumn{2}{|c|}{ Deprived } & \multicolumn{2}{|c|}{ Advantaged } \\
\hline & $M$ & SD & $M$ & SD & $M$ & SD & $M$ & SD \\
\hline \multicolumn{9}{|l|}{ Fairness judgments } \\
\hline Group treatment & 2.67 & 1.14 & 3.95 & 1.20 & 2.94 & 0.93 & 3.75 & 1.39 \\
\hline Personal treatment & 2.11 & 1.12 & 4.38 & 1.14 & 2.49 & 1.09 & 4.24 & 1.15 \\
\hline \multicolumn{9}{|l|}{ Voice intentions } \\
\hline Group treatment & 5.36 & 0.99 & 3.62 & 1.42 & 4.68 & 1.34 & 4.30 & 1.46 \\
\hline Personal treatment & 5.39 & 0.99 & 3.59 & 1.49 & 5.39 & 0.93 & 2.74 & 1.27 \\
\hline
\end{tabular}

Means are on 7-point scales with higher values indicating more positive fairness judgments and stronger voice intentions, respectively

they were brought in a collectivistic mindset; however, they only found it less fair to be deprived than to be advantaged, regardless of the level on which they were treated.

Thus, Study 1 indicated that when participants responded to neutral stimulus materials our Dutch participants found it less fair to be personally deprived than to be group deprived, which is indicative of an individualistic pattern of responses. Furthermore, absent countercultural priming the Dutch participants found it more fair to be personally advantaged than to be group advantaged, which suggests a selfinterested effect. In this way, Study 1 showed that when our Dutch participants were in their default mindset, which we assumed to be individualistic, personal selfinterest affected how fair or unfair they judged the scenarios presented to them.

In contrast, Dutch participants primed with collectivistic mindsets showed strong, robust reactions to our fairness manipulation only, such that they found it less fair to 


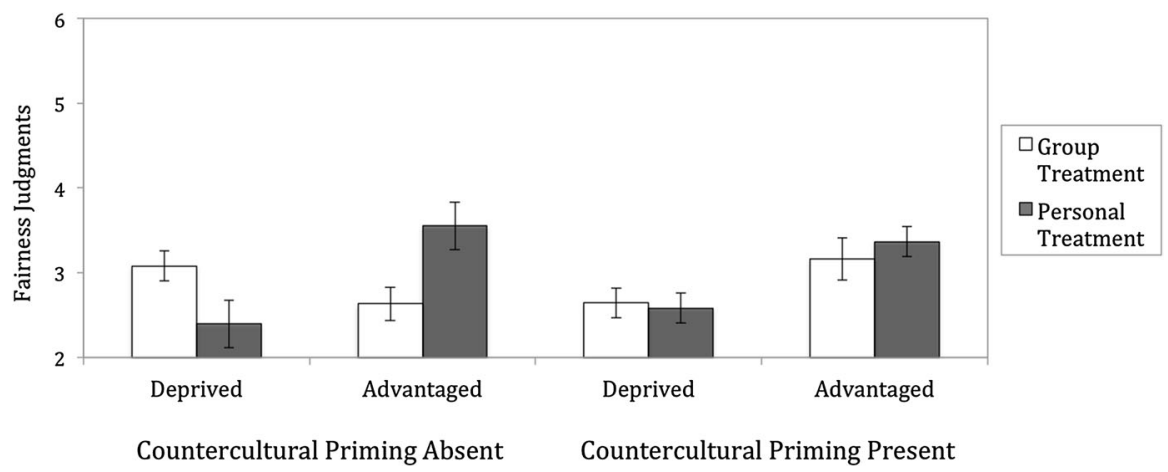

Fig. 1 Fairness judgments among Dutch participants as a function of the absence versus presence of the priming of a collectivistic mindset and being deprived or advantaged as a group or person (Study 1). Error bars represent standard errors of the mean

be deprived than to be advantaged, regardless of whether it was a personal or groupbased disadvantage. This finding suggests that when collectivistic mindsets are salient (in this case by means of experimental priming), this triggers a more inclusive view of the self in which both personal and group treatment matter to people.

We emphasize that we obtained these findings on participants' fairness judgments only. We also note that before strong conclusions are drawn on the basis of these findings, it is important to assess among participants from a collectivistic culture the possible effects of countercultural priming and being deprived or being advantaged as an individual or as a group member. This was the main aim of Study 2.

Study 2

\section{Method}

Participants and Design Two hundred and twenty-nine students at the National University of Singapore in Singapore participated in Study 2 (118 men, 111 women; $\left.M_{\mathrm{age}}=21.93, \mathrm{SD}_{\mathrm{age}}=2.21\right)$. Participants were randomly assigned to one of the conditions of a 2 (countercultural priming: absent vs. present) $\times 2$ (deprivation: deprived vs. advantaged) $\times 2$ (level of treatment: group vs. personal) between subjects design.

Experimental Procedure The method of Study 2 was similar to Study 1 and differed only in the following respects:

Participants of Study 2 were invited to fill out the paper-and-pencil questionnaire in small classroom sessions and received $\mathrm{S} \$ 4$ (approximately $€ 2.50$ ) for their participation.

In Study 2, the condition in which countercultural priming was present to our Singaporean participants was modeled after the individualistic prime of the SDFF 
task (Trafimow et al., 1991). Accordingly, participants in this condition were informed that recent research has shown that people's thoughts about their own distinctiveness tell a lot about them and that we therefore asked them to think about themselves and to complete the following two questions to that effect: (1) "Please think for a minute about what makes you different from your family and friends. How are you a unique being?," and (2) "What do you generally expect yourself to do?" The condition in which countercultural priming was absent was the same as in Study 1.

Study 2 also included the PANAS as a filler task, again yielding reliable scales of positive $(\alpha=.91)$ and negative affective states $(\alpha=.93)$. The content of the scenarios was the same, only the amount of the bonus was converted into Singaporean Dollars. That is, all participants of Study 2 received a year-end bonus of $\$ \$ 850$ (approximately $€ 535$ ) and learned that immediate co-workers or members from other departments received a bonus of either $\mathrm{S} \$ 1500$ (approximately $€ 943$ ) or S\$150 (approximately €94). Participants of Study 2 completed the same set of items as in Study 1, again yielding reliable scales of fairness judgments $(\alpha=.90)$ and intentions to voice opinions $(\alpha=.74)$.

\section{Results}

Fairness Judgments A 2 (countercultural priming) $\times 2$ (deprivation) $\times 2$ (level of treatment) analysis of variance on participants' fairness judgments showed a significant main effect of the deprivation manipulation, $F(1,214)=98.05$, $p<.001, \eta^{2}=.30$. Participants found it less fair to be deprived $(M=2.55$, $\mathrm{SD}=1.10)$ than to be advantaged $(M=4.08, \mathrm{SD}=1.23)$. We also found a twoway interaction effect between deprivation and level of treatment, $F(1,214)=9.65$, $p<.01, \eta^{2}=.03$. This effect indicated that participants found it less fair to be personally deprived $(M=2.29, \mathrm{SD}=1.11)$ than to be group deprived $(M=2.80$, $\mathrm{SD}=1.04), F(1,214)=4.89, p<.03, \eta^{2}=.02$, and that they found it less fair to be group advantaged $(M=3.86, \mathrm{SD}=1.29)$ than to be personally advantaged $(M=4.31, \mathrm{SD}=1.13), F(1,214)=4.33, p<.04, \eta^{2}=.01$. Viewed differently, the effects of the deprivation manipulation were stronger when experienced at the personal level, $F(1,214)=84.69, p<.001, \eta^{2}=.26$, than when experienced at the group level, $F(1,214)=23.49, p<.001, \eta^{2}=.07$. Importantly, the three-way interaction effect between priming, deprivation, and level of treatment was not statistically significant, $F(1,214)=0.01, p>.93, \eta^{2}=.00$. Other effects were also not statistically significant in the $2 \times 2 \times 2$ analysis, $p \mathrm{~s}>.10$.

Voice Intentions A 2 (countercultural priming) $\times 2$ (deprivation) $\times 2$ (level of treatment) analysis of variance on the voice intentions scale yielded a main effect of deprivation, $F(1,214)=94.37, p<.001, \eta^{2}=.28$, an interaction effect between deprivation and treatment, $F(1,214)=11.89, p<.01, \eta^{2}=.04$, and the predicted 


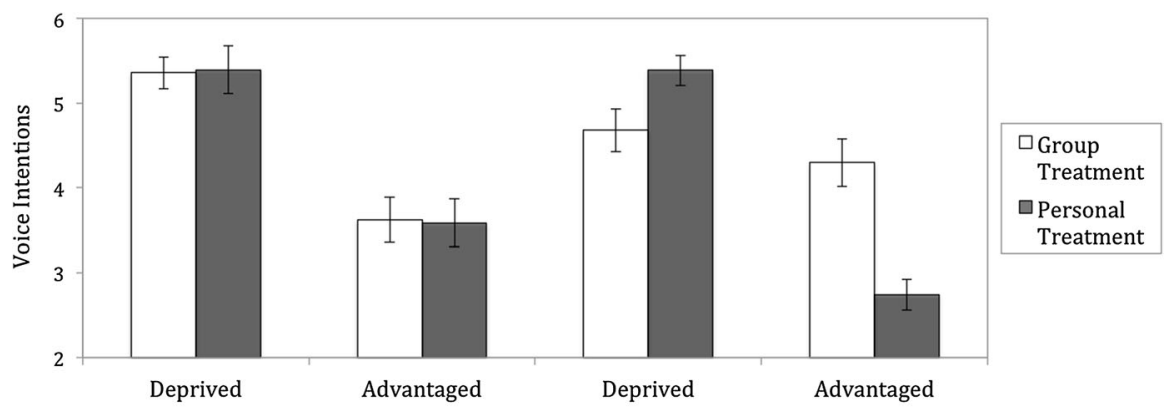

Countercultural Priming Absent Countercultural Priming Present

Fig. 2 Intentions to voice one's opinions among Singaporean participants as a function of the absence versus presence of the priming of an individualistic mindset and being deprived or advantaged as a group or person (Study 2). Error bars represent standard errors of the mean

three-way interaction effect, $F(1,214)=10.50, p<.01, \eta^{2}=.03{ }^{4}$ Other effects were not statistically significant, $p \mathrm{~s}>.20$. Figure 2 illustrates the three-way interaction effect.

In the condition in which countercultural priming was absent the two-way interaction effect between deprivation and level of treatment was not statistically significant, $F(1,214)=.02, p>.88, \eta^{2}=.00$. In this condition, participants showed a main effect of the deprivation manipulation only, $F(1,214)=56.21$, $p<.001, \eta^{2}=.17$, indicating that, in the absence of countercultural priming, our Singaporean participants intended to voice their opinions more against being deprived $(M=5.02, \mathrm{SD}=1.20)$ than against being advantaged $(M=3.52$, $\mathrm{SD}=1.57)$.

In the condition in which countercultural priming was present, we obtained a statistically significant two-way interaction effect between deprivation and level of treatment, $F(1,214)=21.93, p<.001, \eta^{2}=.07$. This effect indicated that, in the presence of countercultural priming, our Singaporean participants intended to voice their opinions somewhat more when they were personally deprived $(M=5.39$, $\mathrm{SD}=0.93)$ than when they were group deprived $(M=4.68, \mathrm{SD}=1.34)$, although we note that this effect was marginally significant only, $F(1,214)=3.68, p=.056$, $\eta^{2}=.01$. Furthermore, when countercultural priming was present, our Singaporean participants reported less intentions to voice their opinions when they were personally advantaged $(M=2.74, \mathrm{SD}=1.27)$ than when they were group advantaged $(M=4.30, \mathrm{SD}=1.46), F(1,214)=20.62, p<.001, \eta^{2}=.06$. Put

\footnotetext{
4 Inspecting Cook's distance measure in this analysis revealed that 7 of the 229 participants of Study 2 (3.1\% of the sample) indicated a distance score of more than 3 SDs above the mean. These participants were excluded from the main analyses of Study 2, leaving a total of 222 participants, with 26-29 participants in each cell of the design. Including the outlying participants in our analyses yielded a significant three-way interaction effect between countercultural priming, deprivation, and level of treatment on participants' intentions to voice their opinions, $F(1,221)=6.97, p<.01, \eta^{2}=.02$, attesting to the robustness of the predicted effect. When controlling for positive and negative affective states, the hypothesized three-way interaction was statistically significant, $F(1,209)=9.78, p<.01, \eta^{2}=.03$, hence the effects of affective states are not reported further in Study 2.
} 
differently, in the presence of countercultural priming, participants showed significantly more intentions to voice their opinions against being personally deprived than against being personally advantaged, $F(1,214)=58.98, p<.001$, $\eta^{2}=.18$, and indicated no significant difference in voice intentions when they were group deprived or group advantaged, $F(1,214)=1.30, p>.25, \eta^{2}=.00$.

\section{Discussion}

The results obtained in Study 2 on participants' voice intentions are in line with what we found in Study 1 on participants' fairness judgments, albeit on a different dependent variable. That is, in Study 2, we found that when experimentally primed with individualistic mindsets our Singaporean participants voiced their opinions somewhat more against being deprived as an individual than against being deprived as a group member (i.e., $p=.056$ ), whereas they intended to voice their opinions less against being advantaged as a person than against being advantaged as a group member. However, when countercultural priming was absent, and hence our Singaporean participants could be assumed to have adopted their normal, collectivistically oriented mindsets, they only had stronger voice intentions when they were deprived than when they were advantaged, independent of the level on which they were treated.

Although the most interesting effects of Study 2 were obtained on a different dependent variable than in Study 1, the findings are noteworthy, we think, and to some extent resemble those obtained in Study 1 in interesting ways. That is, Study 2 showed that in the presence of countercultural priming our Singaporean participants intended to voice their opinions somewhat more against being personally deprived than against being group deprived. This effect was marginally significant only, but the pattern obtained is suggestive of an individualistic kind of effect. Furthermore, when countercultural mindsets were primed the Singaporean participants intended to voice their opinions more against being personally advantaged than against being group advantaged, which indicates a self-interested effect in this condition. Thus, when experimentally primed to view themselves as individuals, Singaporean participants showed individualistic or even self-interested patterns of reactions.

Study 2 also revealed that absent countercultural priming our Singaporean participants showed statistically reliable reactions to the fairness manipulation only such that they intended to voice their opinions more against being deprived than against being advantaged. Thus, in correspondence with at least some findings obtained in Study 1, when participants can be assumed to have adhered to collectivistic mindsets (which was probably the case in Study 2 when no explicit mindsets were made salient and hence our Singaporean participants were likely to default to the values and beliefs that are predominant to their collectivistic culture) fairness issues impacted participants' voice intentions strongly. We again emphasize that the most interesting effects obtained in Studies 1 and 2 were found on different dependent variables. In what follows, we discuss what the two studies have in common and how they differ. 


\section{General Discussion}

Taken together, the two studies presented here show that individualistic and collectivistic mindsets affect reactions to variations in personal and group deprivation. Specifically, people in an individualistic mindset, either because this is embedded in their national culture (which was likely to be the case in the control condition in the Dutch sample of Study 1 in which there was no countercultural priming) or because they are reminded of their personal uniqueness (as was the case in the experimental condition in the Singaporean sample of Study 2 in which countercultural priming was present) respond in more negative terms when they are deprived as an individual person than when they are deprived as a group member and respond in more positive terms when they are advantaged as a person than as a group member. These findings suggest that individualistic or self-interested types of reactions are likely when individualistic mindsets are salient as a result of cultural background (Study 1) and experimental manipulation (Study 2).

Furthermore, both studies also show that when people adhere to collectivistic mindsets, either because of national culture (as in the control condition of the Singaporean sample of Study 2) or because they are experimentally reminded of their similarities with family and friends (as was the case in the countercultural condition in the Dutch sample of Study 1), they respond more negatively to being deprived than to being advantaged, irrespective of whether this manipulation affected them as individuals or as group members. One way to interpret these findings is to conclude that in our collectivistic mindset conditions (derived by experimental manipulation or national background), we observed reactions that are indicative of a more inclusive view of the self in which both personal and group treatment matter to participants. This finding needs to be supported in future research. Future research is also needed to deepen our understanding of this finding. This brings us to a discussion of the implications as well as the limitations of the findings presented here.

\section{Implications}

With respect to implications for relative deprivation theory, the results presented provide an explanation for why people sometimes may be more sensitive to situations in which they are personally deprived, whereas in other circumstances they respond more strongly to being group deprived. It might well be the inclination to think in terms of "I" or to think in terms of "we" can explain these differential responses to personal and group deprivation. Thereby the present research builds on earlier research that linked personal and group deprivation to salient social identities (Ellemers, 2001; Kawakami \& Dion, 1993; Smith et al., 1994, 2012). That is, people from individualistic countries or who have adopted individualistic mindsets in some other ways are more likely to respond to variations in personal deprivation than to variations in group deprivation. People with a collectivistic background or who have adopted a collectivistic mindset, pay attention to general disadvantage or advantage and do not appear to be sensitive to variations in being treated as an individual or a group member. Perhaps this latter finding suggests that an inclusive view of the self 
in which both personal and group treatment matter is especially prevalent under conditions or in cultures in which collectivistic norms and values are important and/ or salient. This suggestion needs further empirical examination.

We further note that even though researchers all over the world have drawn upon relative deprivation, they often use it to construct post hoc explanations of unexpected findings, and data reported are often correlational and rarely involve direct cross-national comparisons (see, e.g., the Smith et al., 2012, meta-analysis). There is a growing realization that research findings obtained in one culture cannot always be extrapolated easily to other cultures and that appropriate attention to cross-cultural differences is pivotal for any reliable social and behavioral science, including the scientific study of social justice and relative deprivation. In the current paper, we employed an experimental approach to cross-cultural research on relative deprivation using a method in which we primed (vs. did not prime) countercultural mindsets among people from individualistic and collectivistic cultures, and we assessed how the resulting variations in culture (i.e., country) and context (i.e., experimental condition) impacted participants' reactions to being deprived or advantaged as an individual person or as a member of a group.

One implication that follows from the two experiments presented here is that by means of countercultural priming we may gain insight into the psychological dimensions that account for cross-cultural differences in how people treat relative deprivation. In particular, what the current studies show is that identifying countries or cultures that differ reliably and meaningfully on the individualism-collectivism dimension (e.g., Hofstede, 2001), and then randomly assigning some of the research participants to conditions that prime norms and values that contradict what is prevalent in their culture and assigning other participants to conditions in which they are likely to draw upon prevalent cultural norms can yield research results that help to interpret reactions to personal and group deprivation from members of individualistic and collectivistic cultures. The results thus obtained have a high level of internal validity due to the reliance on experimental manipulations. As noted, this is important because a recurring issue in the study of cross-cultural differences is trying to get a good handle on potential problems of convenience sampling and lack of insight into underlying psychological processes. The method of experimental countercultural priming may help to overcome at least some of these problems.

The finding that it is possible to push people in a countercultural direction provides support for the idea, that cultural norms are malleable, and thereby for the possibility of priming countercultural norms (e.g., Oyserman, 2011; Oyserman \& Lee, 2008; Van den Bos et al., 2010, 2013). One reason why this insight is important is because it allows for studying cross-cultural differences with experimental control. As noted, we think there are good reasons why it is preferable, when possible, to manipulate the cultural dimensions under consideration, so that causal relationships can be inferred (Spencer et al., 2005; Van den Bos et al., 2010, 2013). The present research shows that this is possible in cross-cultural research even when complex social behavioral patterns are targeted. Still, to gain more solid ground, the countercultural priming paradigm should be extended to other cultural dimensions and different behavioral patterns. 


\section{Limitations}

We note explicitly that the two studies reported are not without flaws. For example, we had no manipulation check or pilot data to confirm that the individualistic and collectivist primes worked. However, we carefully constructed our stimulus materials on the basis of thorough conceptualization of cross-cultural differences in individualism and collectivism (see Trafimow et al., 1991). Furthermore, peopleactivated mindsets might be difficult to measure reliably without raising concerns of suspicion or performance demands among participants. That noted, it clearly would be desirable to have more definitive evidence that the results emerged for the reasons delineated in our line of reasoning.

Furthermore, based on the work by Trafimow et al. (1991) and Hofstede (2001), we assumed that in the control conditions of both studies, our student participants in the Netherlands emphasized values associated with individualism, whereas those in Singapore assigned more importance to values associated with collectivism. Whereas the results we obtained are consistent with this possibility, further research clearly is needed to evaluate these assumptions. The Netherlands and Singapore probably differ on more cultural dimensions than individualism-collectivism alone (see, e.g., Hofstede, 2001), and it is important for future research to evaluate whether any of the present findings may be accounted for by other underlying mechanisms and psychological processes.

Another possible limitation is that research participants need to be susceptible to the mindset induction that we operationalized in the current studies by having participants respond to two open-ended questions. Careful construction of the words used in the questions and instructions is pivotal. We have some good experiences with these kinds of manipulations (see also Van den Bos et al., 2010, 2013), but nevertheless an important prerequisite is that participants should be able and willing to pick up the meaning conveyed in the stimulus materials. Future researchers should be aware of this issue as well as the need to pretest stimulus materials in their samples.

The exact pattern of effects obtained also needs to be discussed. That is, whereas we found in both our experiments that individualistic mindsets (whether default by national culture or induced by means of experimental manipulation) led to particularly strong reactions to variations in personal deprivation, collectivistic mindsets did not lead to sensitivity to variations in group deprivation only or primarily. Thus, collectivistic mindsets (whether default by culture or induced by manipulation) led to particularly strong reactions to the manipulation that varied whether participants were deprived or advantaged and this was found both when participants were affected as individuals and as group members. Perhaps these findings imply that whereas individualistic mindsets lead people to be oriented to what happens to themselves primarily, collectivistic mindsets trigger a more inclusive view of the self, in which both personal treatment and group treatment matter to people. This possible implication should be treated with caution, of course, in part because the current studies did not assess participants' self-concepts directly. Nevertheless, in light of the interesting debate about pan-cultural self-enhancement and the role that self-concepts play in possible cross-cultural differences (see, e.g., 
Heine et al., 1999; Sedikides et al., 2003), it would be interesting to examine the content of self-concepts pertaining to relative deprivation issues using the countercultural priming paradigm.

We further note that in the present studies all our participants responded to unfair outcome distributions. The only thing that we varied was whether the unfairness was to the disadvantage or to the advantage of the participants involved. We thought inclusion of the advantaged condition was important because this allowed us to assess self-interested responses among our participants (see, e.g., Van den Bos, Peters, Bobocel, \& Ybema, 2006). That is, whereas more negative responses to personal deprivation indicate personal or individualistic kind of reactions, more positive reactions to personal advantageous outcomes suggest self-centered or selfinterested types of responses. In this way, the findings of our two studies yield evidence for both individualistic and self-interested effects. Thus, inclusion of both deprived and advantaged conditions seemed important, but we hasten to note that other instances of relative deprivation, other fairness issues, and other control conditions should be included in future research.

Perhaps inclusion of an additional control condition in which the default cultural mindset is made salient (such as priming individualism when conducting research in the Netherlands and collectivism when studying reactions in Singapore) might be worthwhile when using the countercultural priming paradigm in future research. We note that although this extra condition was now absent in the present studies, we still were able to contrast conditions in which participants could be assumed to adhere to their default cultural values with conditions in which they adhered to values that are opposite to what is known to be prevalent in their national culture (see, e.g., Hofstede, 2001; Trafimow et al., 1991).

Perhaps more importantly, precisely because of its default or non-salient quality, a default cultural norm is often in the back of conscious awareness and people rarely think of it explicitly (Kitayama, 2002). Hence, bringing the cultural norm into awareness may change the effects that the norm normally has on people's reactions. For instance, reminding participants from individualistic cultures of their personal uniqueness may lead them to respond more strongly to personal deprivation than they normally tend to do. And priming similarities and differences with family and friends among members from a collectivistic culture may lead to more group-oriented reactions than we now found in our control conditions. Moreover, it is also possible that priming pro-cultural norms could attenuate the effects of cultural mindsets on sensitivity to personal and group deprivation. For example, through conscious reflection on individualistic norms, Dutch participants might conclude that it is actually not that good to be an individualist and try to distance themselves from individualistic values (see also Van den Bos et al., 2013). In short, we think that by comparing experimental conditions, in which countercultural norms were primed with control conditions in which participants completed two questions that were shown in earlier studies to be neutral and in which participants could be assumed to adhere to their default cultural values, we focused on the most relevant contrast for the current purposes of being the first to use a countercultural-experimental approach to the study of relative deprivation and individualism-collectivism. That noted, we would applaud if different control conditions would be used in future research. 
We also note that we analyzed the Dutch and Singaporean samples separately, even though the samples responded to the same stimulus materials. One of the reasons why we did this was that the Dutch and the Singaporean samples are different in essence. This implied that the participants were drawn from different populations, were tested under different circumstances, and different sampling methods were used. Therefore, we think it is methodologically more appropriate to subject them to statistical analyses as two samples.

Finally, we also have seen some apparent issues with participants from different cultures or different samples being differentially sensitive to different types of dependent variables. This is not an uncommon observation in cross-cultural studies (e.g., Matsumoto, 2003). We again emphasize that in both our studies participants were presented with 24 items assessing different categories of participant reactions (i.e., fairness judgments, intentions to criticize and voice opinions, and positive and negative feelings; see Van Veldhuizen, 2013). To construct meaningful scales from these variables, we started with exploratory analyses in our two studies to assess which of these variables triggered the strongest response patterns in terms of our hypotheses in each of the samples. In this way, we computed two scales, one pertaining to fairness judgments and one regarding intentions to voice one's opinions, and we submitted those scales to subsequent analyses reported here. In this way, we think we provided the most informative story in terms of testing our hypotheses in the two samples identified. In particular, we were able to show that our two samples responded differently to the two scales reported. We deliberately chose to use this method, because we believe that as social psychologists we should not aim to abstract peoples' responses down to one single emotion, thought, or behavior that is assumed to be universal or equally sensitive across different samples. It is not realistic to think that you can always predict how and on what response dimension people will respond exactly, especially not when you study cross-cultural samples that differ in various important ways. This said, we stress that future research is needed to test for the robustness of the effects reported in the present paper. Such future studies also would do well to include non-hypothetical instances of personal and group deprivation.

\section{Coda}

To conclude, the current paper presented a countercultural priming approach to the study of cross-cultural differences. This approach was illustrated by an examination of responses to personal and group deprivation in the Netherlands and Singapore, among participants for whom countercultural mindsets were primed or not. The findings presented suggest that people in individualistic mindsets are primarily focused on their personal state of affairs; they are more sensitive to being personally deprived than to being collectively deprived. People in collectivistic mindsets, in contrast, seem to be focused on both their personal state of affairs and on the position of the group to which they belong. Consequently, they respond in similar ways to personal and group deprivation. Importantly, these response patterns are independent of whether the cultural mindset belongs to one's national culture or is temporarily made salient through contextual priming. This implies that 
when cultural backgrounds impact people's deprivation responses in important ways. That noted, the findings presented also reveal that cultural mindsets are not fixed and are malleable by social context. Taken together, these findings suggest important cultural and contextual differences pertaining to personal and group deprivation.

\section{References}

Baumeister, R. F. (2005). The cultural animal: Human nature, meaning, and social life. New York: Oxford University Press.

Bechtoldt, M. N., Choi, H. S., \& Nijstad, B. A. (2012). Individuals in mind, mates by heart: Individualistic self-construal and collective value orientation as predictors of group creativity. Journal of Experimental Social Psychology, 48, 838-844.

Bechtoldt, M. N., De Dreu, C. K., Nijstad, B. A., \& Choi, H. S. (2010). Motivated information processing, social tuning, and group creativity. Journal of Personality and Social Psychology, 99, 622-637.

Brockner, J. (2003). Unpacking country effects: On the need to operationalize the psychological determinants of cross-national differences. Research in Organizational Behavior, 25, 333-367.

Cohen, J., Cohen, P., West, S. G., \& Aiken, L. S. (2003). Applied multiple regression/correlation analysis for the behavioral sciences (3rd ed.). Hillsdale, NJ: Erlbaum.

Cook, R. D. (1977). Detection of influential observations in linear regression. Technometrics, 19, 15-18.

Crosby, F. (1976). A model of egoistical relative deprivation. Psychological Review, 83, 85-112.

Dambrun, M., Taylor, D. M., McDonald, D. A., Crush, J., \& Méot, A. (2006). The relative deprivationgratification continuum and the attitudes of South Africans toward immigrants: A test of the V-curve hypothesis. Journal of Personality and Social Psychology, 91, 1032-1044.

Ellemers, N. (2001). Social identity and relative deprivation. In I. Walker \& H. J. Smith (Eds.), Relative deprivation: Specification, development and integration (pp. 239-264). New York: Cambridge University Press.

Greenberg, J., Solomon, S., \& Pyszczynski, T. (1997). Terror management theory of self-esteem and cultural worldviews: Empirical assessments and conceptual refinements. In M. P. Zanna (Ed.), Advances in experimental social psychology (Vol. 29, pp. 61-139). New York: Academic Press.

Guimond, S., \& Dambrun, M. (2002). When prosperity breeds intergroup hostility: The effects of relative deprivation and relative gratification on prejudice. Personality and Social Psychology Bulletin, 28, 900-912.

Heine, S. J., Markus, H. R., Lehman, D. R., \& Kitayama, S. (1999). Is there a universal need for positive self-regard? Psychological Review, 106, 766-794.

Hofstede Centre. (2013). National culture: Countries. Retrieved December 15, 2014 from http://geerthofstede.com/countries.html.

Hofstede, G. (2001). Culture's consequences: Comparing values, behaviors, institutions and organizations across nations (2nd ed.). Thousand Oaks, CA: Sage Publications.

Hong, Y. (2009). A dynamic constructivist approach to culture. Moving from describing culture to explaining culture. In R. S. Wyer, C. Chiu, \& Y. Hong (Eds.), Understanding culture: Theory, research, and application (pp. 3-23). New York: Taylor \& Francis Group.

Hong, Y., \& Chiu, C. (2001). Toward a paradigm shift: From cultural differences in social cognition to social cognitive mediation of cultural differences. Social Cognition, 19, 118-196.

Hui, C. H., \& Triandis, H. C. (1985). Measurement in cross-cultural psychology: A review and comparison of strategies. Journal of Cross-Cultural Psychology, 16, 131-152.

Kawakami, K., \& Dion, K. (1993). The impact of salient self-identities on relative deprivation and action intentions. European Journal of Social Psychology, 23, 525-540.

Kitayama, S. (2002). Culture and basic psychological processes: Toward a system view of cultureComment on Oyserman et al. (2002). Psychological Bulletin, 128, 89-96.

Kühnen, U., Hannover, B., \& Schubert, B. (2001). The semantic procedural-interface model of the self: the role of self-knowledge for context-dependent versus context-independent modes of thinking. Journal of Personality and Social Psychology, 80, 397-409. 
Kühnen, U., \& Oyserman, D. (2002). Thinking about the self influences thinking in general: Cognitive consequences of salient self-concept. Journal of Experimental Social Psychology, 38, 492-499.

Loseman, A., Miedema, J., Van den Bos, K., \& Vermunt, R. (2009). Exploring how people respond to conflicts between self-interest and fairness: The influence of threats to the self on affective reactions to advantageous inequity. Australian Journal of Psychology, 61, 13-21.

Matsumoto, D. (2003). Cross-cultural research. In S. Davis (Ed.), The handbook of research methods in experimental psychology (pp. 189-208). Oxford, UK: Blackwell.

Matsumoto, D., \& Hee Yoo, S. (2006). Toward a new generation of cross-cultural research. Perspectives on Psychological Science, 1, 234-250.

Miller, J. G. (2002). Bringing culture to basic psychological theory: Beyond individualism and collectivism-Comment on Oyserman et al. (2002). Psychological Bulletin, 128, 97-109.

Moscatelli, S., Albarello, F., Prati, F., \& Rubini, M. (2014). Badly off or better off than them? The impact of relative deprivation and relative gratification on intergroup discrimination. Journal of Personality and Social Psychology, 107, 248-264.

Nisbett, R. E., \& Ross, L. (1980). Human inference: Strategies and shortcomings of social judgment. Englewood Cliffs, NJ: Prentice-Hall.

Oyserman, D. (2011). Culture as situated cognition: Cultural mindsets, cultural fluency, and meaning making. In W. Stroebe \& M. Hewstone (Eds.), European review of social psychology (Vol. 22, pp. 164-214). Oxon, UK: Psychology Press.

Oyserman, D., Coon, H. M., \& Kemmelmeier, M. (2002). Rethinking individualism and collectivism: Evaluation of theoretical assumptions and meta-analyses. Psychological Bulletin, 128, 3-72.

Oyserman, D., \& Lee, S. W. (2007). Priming "culture": Culture as situated cognition. In S. Kitayama \& D. Cohen (Eds.), Handbook of cultural psychology (pp. 255-276). New York: Guilford Press.

Oyserman, D., \& Lee, S. W. (2008). Does culture influence what and how we think? Effects of priming individualism and collectivism. Psychological Bulletin, 134, 311-342.

Runciman, W. G. (1996). Relative deprivation and social justice: A study of attitudes to social inequality in twentieth-century England. Berkeley: University of California Press.

Sedikides, C., Gaertner, L., \& Toguchi, Y. (2003). Pancultural self-enhancement. Journal of Personality and Social Psychology, 84, 60-79.

Simmons, J. P., Nelson, L. D., \& Simonsohn, U. (2012). A 21-word solution. Dialogue, 26, 4-7.

Smith, H. J., \& Ortiz, D. J. (2001). Is it just me? The different consequences of personal and group relative deprivation. In I. Walker \& H. J. Smith (Eds.), Relative deprivation: Specification, development and integration (pp. 91-115). New York: Cambridge University Press.

Smith, H. J., Pettigrew, T. F., Pippin, G. M., \& Bialosiewicz, S. (2012). Relative deprivation: A theoretical and meta-analytic review. Personality and Social Psychology Review, 16, 203-232.

Smith, H. J., Spears, R., \& Oyen, M. (1994). "People like us": The influence of personal deprivation and group membership salience on justice evaluations. Journal of Experimental Social Psychology, 30, 277-299.

Spencer, S. J., Zanna, M. P., \& Fong, G. T. (2005). Establishing a causal chain: Why experiments are often more effective than mediational analyses in examining psychological processes. Journal of Personality and Social Psychology, 89, 845-851.

Stouffer, S. A., Suchman, E. A., DeVinney, L. C., Star, S. A., \& Williams, R. M. (1949). The American soldier: Adjustment during Army life (Vol. 1). Princeton: Princeton University Press.

Trafimow, D., Triandis, H. C., \& Goto, S. G. (1991). Some tests of the distinction between the private self and the collective self. Journal of Personality and Social Psychology, 60, 649-655.

Triandis, H. C., McCusker, C., \& Hui, C. H. (1990). Multimethod probes of individualism and collectivism. Journal of Personality and Social Psychology, 59, 1006-1020.

Van den Bos, K. (2001). Uncertainty management: The influence of uncertainty salience on reactions to perceived procedural fairness. Journal of Personality and Social Psychology, 80, 931-941.

Van den Bos, K., Brockner, J., Stein, J. H., Steiner, D. D., Van Yperen, N. W., \& Dekker, D. M. (2010). The psychology of voice and performance capabilities in masculine and feminine cultures and contexts. Journal of Personality and Social Psychology, 99, 638-648.

Van den Bos, K., Brockner, J., Van den Oudenalder, M., Kamble, S. V., \& Nasabi, A. (2013). Delineating a method to study cross-cultural differences with experimental control: Tthe voice effect and countercultural contexts regarding power distance. Journal of Experimental Social Psychology, 49, 624-634.

Van den Bos, K., Buurman, J., De Theije, V., Doosje, B., Loseman, A., Van Laarhoven, D., et al. (2012). On shielding from death as an important yet malleable motive of worldview defense: Christian 
versus Muslim beliefs modulating the self-threat of mortality salience. Social Cognition, 30, $778-802$.

Van den Bos, K., \& Miedema, J. (2000). Toward understanding why fairness matters: The influence of mortality salience on reactions to procedural fairness. Journal of Personality and Social Psychology, 79, 355-366.

Van den Bos, K., Peters, S. L., Bobocel, D. R., \& Ybema, J. F. (2006). On preferences and doing the right thing: Satisfaction with advantageous inequity when cognitive processing is limited. Journal of Experimental Social Psychology, 42, 273-289.

Van Prooijen, J.-W., Van den Bos, K., \& Wilke, H. A. M. (2002). Procedural justice and status: Status salience as antecedent of the fair process effect. Journal of Personality and Social Psychology, 83, 1353-1361.

Van Veldhuizen, T. S. (2013). Cultural and contextual differences pertaining to relative deprivation: Collectivistic and individualistic responses. MSc thesis, Utrecht University.

Walker, I. (1999). Effects of personal and group relative deprivation on personal and collective selfesteem. Group Processes and Intergroup Relations, 2, 365-380.

Walker, I., \& Mann, L. (1987). Unemployment, relative deprivation and social protest. Personality and Social Psychology Bulletin, 13, 175-283.

Watson, D., Clark, L. A., \& Tellegen, A. (1988). Development and validation of brief measures of positive and negative affect: The PANAS scales. Journal of Personality and Social Psychology, 54, 1063-1070. 